\title{
QUANTUM SENSING
}

WITH NITROGEN-VACANCY

COLOUR CENTERS IN DIAMOND

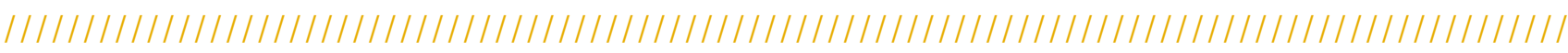

\section{Thierry DEBUISSCHERT}

Thales Research \& Technology, 1 avenue Augustin Fresnel, 91767 Palaiseau cedex, France.

*thierry.debuisschert@thalesgroup.com

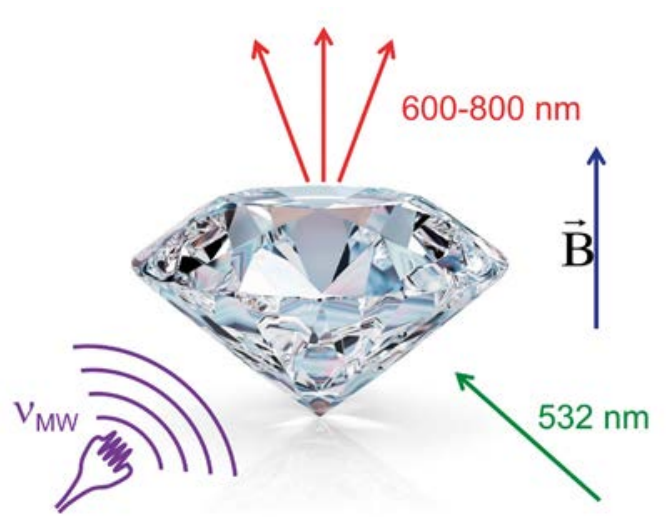

https://doi.org/10.1051/photon/202110750
Quantum sensing exploits the possibility of manipulating single quantum objects and of measuring external physical quantities with unprecedented accuracy. It offers new functionalities that cannot be obtained with classical means. Quantum sensors can be based on atomic vapours, cold atoms, dopants in solid-state materials, etc. In the latter category, the nitrogen vacancy centre in diamond has received particular attention in recent years due to its very attractive characteristics.

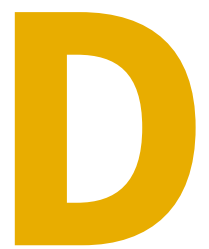

iamond is a beautiful and well-known jewel. Its ability to trap visible light when properly cut and polished yields its distinctive flashing appearance. Diamond is a crystal made only of carbon atoms, which has many appealing properties. It has excellent transparency across the entire visible spectrum; it is one of the hardest materials known with excellent mechanical properties; it has excellent thermal conductivity with very efficient heat dissipation. It is therefore widely used in industry, and the large-scale production of artificial diamond has motivated a great deal of effort on the part of crystal producers. The first technique consisted of reproducing in growth chambers conditions similar to those existing in the heart of volcanoes. Under high pressure and high temperature, carbon atoms can form diamond crystals similar to natural crystals. However, they generally contain too many impurities for applications in the field of sensing or electronics. In the 2000s, a new growth technique was developed, based on plasma-assisted chemical vapour deposition. Here, carbon atoms are deposited layer by layer, resulting in an almost perfect single crystal with a very low level of impurities. Large diamonds of a few millimetres in size and a few hundred micrometres in thickness can be produced with excellent properties. Artificial diamonds are therefore the ideal material for a large number of new applications. For example, these ultra-pure crystals can be doped in a controlled manner with different species to modify their electronic properties.

Natural diamonds can have defects that give them specific colours and are therefore called colour 
centres. One of them, the nitrogen vacancy $(\mathrm{NV})$ centre, produces a red luminescence that yields a pink colour. In a perfect diamond lattice, a nitrogen (N) atom is substituted for a carbon (C) atom. A missing carbon nearby creates a vacancy $(\mathrm{V})$. The nitrogen and the vacancy form what is called the nitrogen vacancy centre (NV). Nitrogen vacancy centres can exist as isolated colour centres in the diamond. A first application in quantum technology was to use them as single photon emitters. Such single photons can then be used to perform quantum cryptography experiments to establish a secret key between two parties [1].

\section{BASICS}

To better understand the properties of the nitrogen vacancy centre, it is necessary to study its atomic structure
(See Fig. 1). The NV centre can exist under different states of charge. The neutral state $N V^{0}$ has an unpaired electron. The negatively charged state $N V$ - captures an additional electron and therefore has two unpaired electrons. The latter is of particular interest for quantum sensing and we will only consider this one, now referred to as NV for the sake of simplicity. The NV centre is similar to an atom nestled in a solid-state matrix. It has energy levels with well-defined spin properties which naturally couple to the magnetic field. In typical experiments, the NV centre is optically pumped, usually with a green laser beam at a wavelength of $532 \mathrm{~nm}$. This induces optical transitions between ground and excited states, resulting in the emission of red fluorescence around $637 \mathrm{~nm}$. As a result of this process, the $\mathrm{NV}$ centre resides in its lowest

Figure 1. Figure 1. (a) The NV center of diamond is constituted by a nitrogen atom (N) substituted to a carbon atom, and a vacancy in an adjacent site. This quantum object absorbs light in the green (at $532 \mathrm{~nm}$ in our case) and emits a perfectly stable photoluminescence in the red domain (between 600 to $800 \mathrm{~nm}$ ).

(b) Energetic diagram associated to the internal electronic spin of the negatively charged NV center. The degeneracy between the state of zero spin $\left(m_{s}=0\right)$ and the states of non-zero spin $\left(m_{s}= \pm 1\right)$ is lifted by the spin-spin interaction. The presence of an external magnetic field (in blue), lifts the degeneracy between the states $m_{s}=-1$ and $m_{s}=+1$ through the Zeeman effect. The frequency difference is proportional to the projection of the magnetic field on the NV axis, $B_{\mathrm{NV}}$.

(c) Electron Spin Resonances (ESR) spectrum. Resonances between the state $m_{s}=0$ and the states $m_{s}= \pm 1$ induced by a microwave field (in purple) can be detected optically by a decrease of the photoluminescence intensity. The frequency difference between the two resonances is directly related to the value of $B_{\mathrm{NV}}$. (taken from A. Nowodzinski et al., Microelectronics Reliability 55 (2015) 1549-1553)

a)

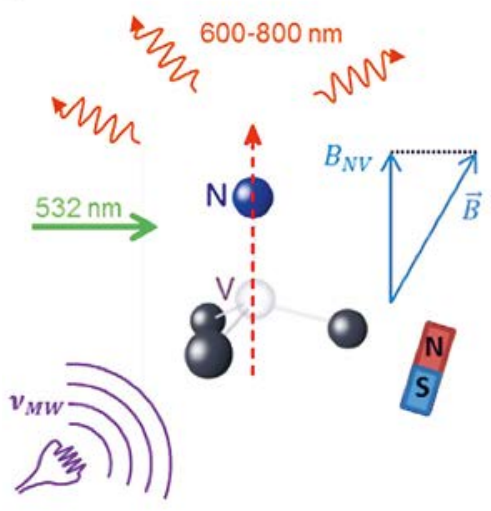

b)
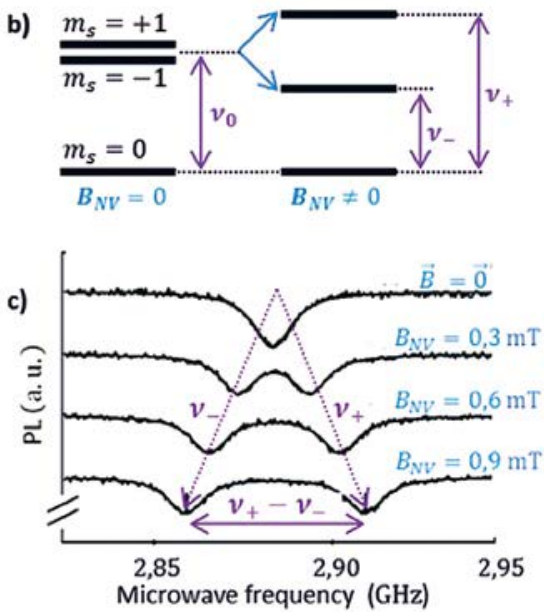

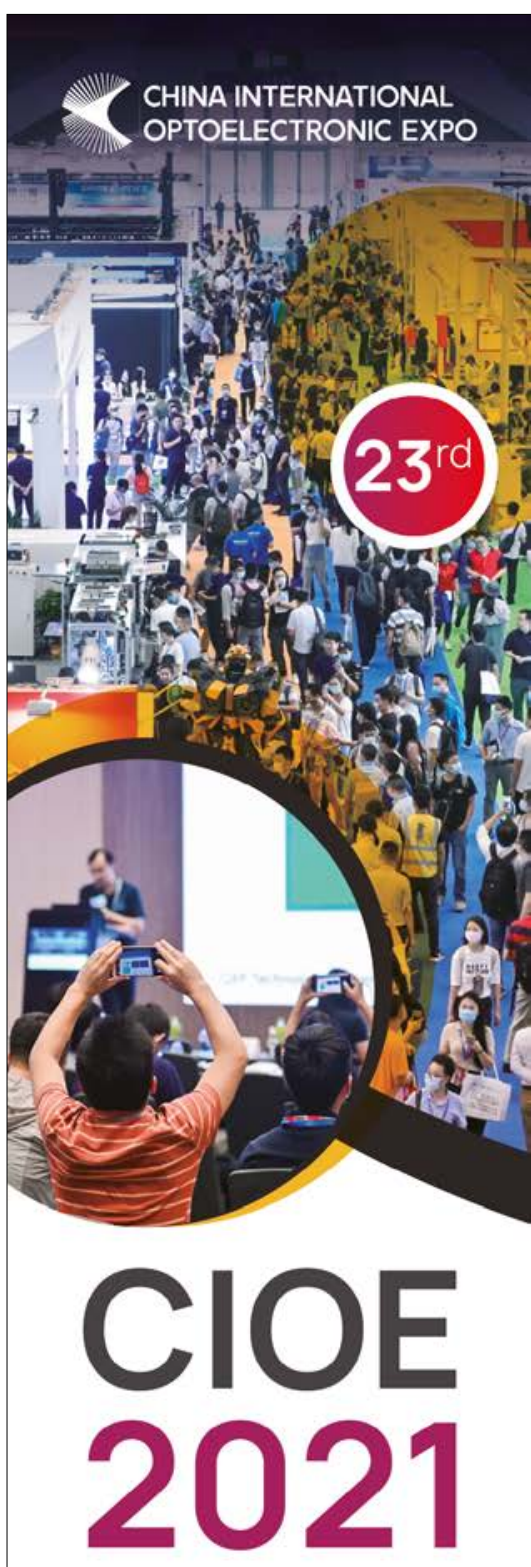

WORLD'S LEADING OPTOELECTRONIC EVENT

SEPTEMBER 1-3, 2021 Shenzhen World Exhibition \& Convention Center

EXHIBITION AREA: $160,000 \mathrm{M}^{2}$ EXHIBITORS: $3,000+$ VISITORS: $95,000+$

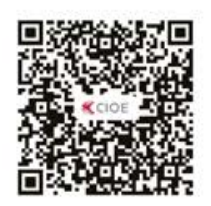

$\bigoplus$ WWW.CIOE.CN/EN 


\section{the NV centre in diamond is similar to an atomic size magnet capable of measuring an applied magnetic field with nanometer scale resolution.}

energy level. The NV centre is therefore polarized in a well-defined quantum state. A similar polarization can be achieved when cooling the sample to cryogenic temperature. Here, however, neither cryostat nor heavy equipment is required and the NV centre can be used under ambient conditions in a standard laboratory environment. This is a major advantage over other techniques and it greatly facilitates the use of NV centres outside the laboratory for a variety of applications. Being in a well-defined quantum state, the NV centre can then be coherently manipulated as an elementary quantum object. This is at the heart of the so-called second quantum revolution. The energy difference between the levels of lowest energy corresponds to a frequency of 2.87 GHz. Applying a microwave radiation at this frequency induces a resonant transition between those levels. Therefore, the NV center, initially in the lowest energy level, is now partly in a higher energy level. This results in a decrease of the fluorescence intensity that can be explained by the properties of the transitions between the energy levels. By sweeping the microwave frequency, a decrease in the fluorescence spectrum can be observed which indicates the transition from ground level to higher levels, very similar to what happens with ordinary atoms. This Optical Detection of Magnetic Resonance (ODMR) is the main property of the NV centre used as a quantum sensor [2].

To go further with the properties of NV centres, it should be noted that the levels involved in the transitions have a well-defined spin state, i.e. an intrinsic magnetic moment that can couple with an external magnetic field. Consequently, the application of an external magnetic field induces a shift in the energy levels (Zeeman shift) which results in a change in the frequencies of the magnetic resonance transitions. By measuring those frequencies, the value of the applied magnetic field in the direction of the $\mathrm{N}-\mathrm{V}$ axis can be obtained directly. In addition, the use of an ensemble of NV centres makes it possible to measure both the magnitude and direction of the magnetic field. Therefore, the NV centre in diamond is similar to an atomic size magnet capable of measuring an applied magnetic field with nanometer scale resolution. Other physical quantities can be measured with $\mathrm{NV}$
Figure 2: Example of a scanning NV magnetometer. A diamond tip with a single NV center at its end is held by an AFM tuning fork. The NV center is excited by a green pump laser. The NV fluorescence is guided by the tip acting as an optical waveguide and then collected by a microscope objective. Measuring the level of luminescence allows retrieving the magnetic field value below the tip. The magnetization distribution is measured with a spatial resolution of $50 \mathrm{~nm}$ when scanning the tip over the magnetic sample. (Courtesy of the Quantum Sensing Group, University of Basel) 
centres. For example, the application of pressure or thermal heating causes a change in crystal lattice size which can induce a shift in the resonances of the $\mathrm{NV}$ centre. This gives access to the measurement of these quantities.

\section{APPLICATIONS IN SENSING}

The versatile sensing capabilities of $\mathrm{NV}$ centres, combined with their simple operating conditions, make them very attractive for applications in various fields such as the automotive industry, communications or medical applications. This is why European research groups in this field launched the European project ASTERIQS whose objective is to develop new sensors with optimal sensitivity and resolution that exploit the quantum properties of nitrogen vacancy centres in diamond. The first example is NV scanning magnetometry (see Fig. 2). A diamond tip with a single NV centre located at its end is positioned at the end of an AFM tuning fork. Scanning the tip a few tens of nanometres above a magnetic sample allows its structure to be recovered with a typical spatial resolution of $50 \mathrm{~nm}$. This is a new tool that makes it possible to study structures such as antiferromagnetic domains with unprecedented resolutions and opens up entirely new fields in spintronics and nanomagnetism. This research is being carried out by several laboratories including the CNRS, ETH Zurich and the University of Basel [3]. This technology is currently being transferred to startups such as QNAMI and QZABRE.

In a second example, $\mathrm{NV}$ centres are used to monitor the behaviour of matter under high pressure (see Fig. 3). The high pressure is achieved in a cell consisting of two diamond anvils which are pressed against each other. One of these anvils contains NV centres near its surface which monitor the magnetic field in the cell. This is a new tool for studying the transition of a material from its normal phase to its superconducting phase under high pressure, which results in a change in its magnetic properties [4]. This work carried out by ENS Paris-Saclay has been transferred to Attocube in order to develop an instrument capable of making measurements under high pressure and at cryogenic temperatures.

The EC Quantum Flagship program [5] targets the development of a European industry in the field of quantum technologies. Several companies are active at different stages of the value chain. Element 6 is now capable of producing large "quantum grade" diamond samples with very low residual stress and a high concentration of NV centres of the order of a few ppm; this is necessary to develop high-sensitivity sensors. Attocube is developing new platforms that will enable magnetic materials to be characterised at the nanometre scale and at cryogenic temperature. Thales is developing a NV-based spectrum analyser that converts microwave frequencies into an optical signal. It can instantaneously monitor a wide spectrum of frequencies, which is necessary for recent developments in the field of communications, such as 5G and cognitive radio, or for radar applications over a wide frequency band. Bosch is targeting the electric car market. It is developing a magnetometer with a miniaturised NV-based sensor head that is capable of monitoring the electric current in a car battery with both high dynamics and high sensitivity.

NV centres offer many other application perspectives. Significant progress is being made in the analysis of the chemical structure of a single molecule. To do this, the molecule rests on the surface of a diamond crystal containing $\bullet \bullet$

\section{This is a new tool for studying the transition of a material from its normal phase to its superconducting phase under high pressure, which results in a change in its magnetic properties}

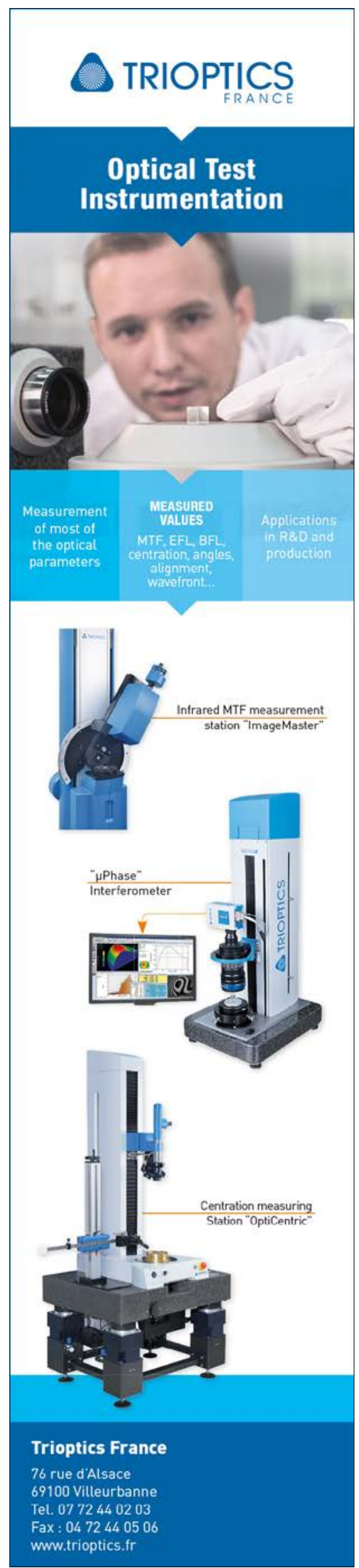




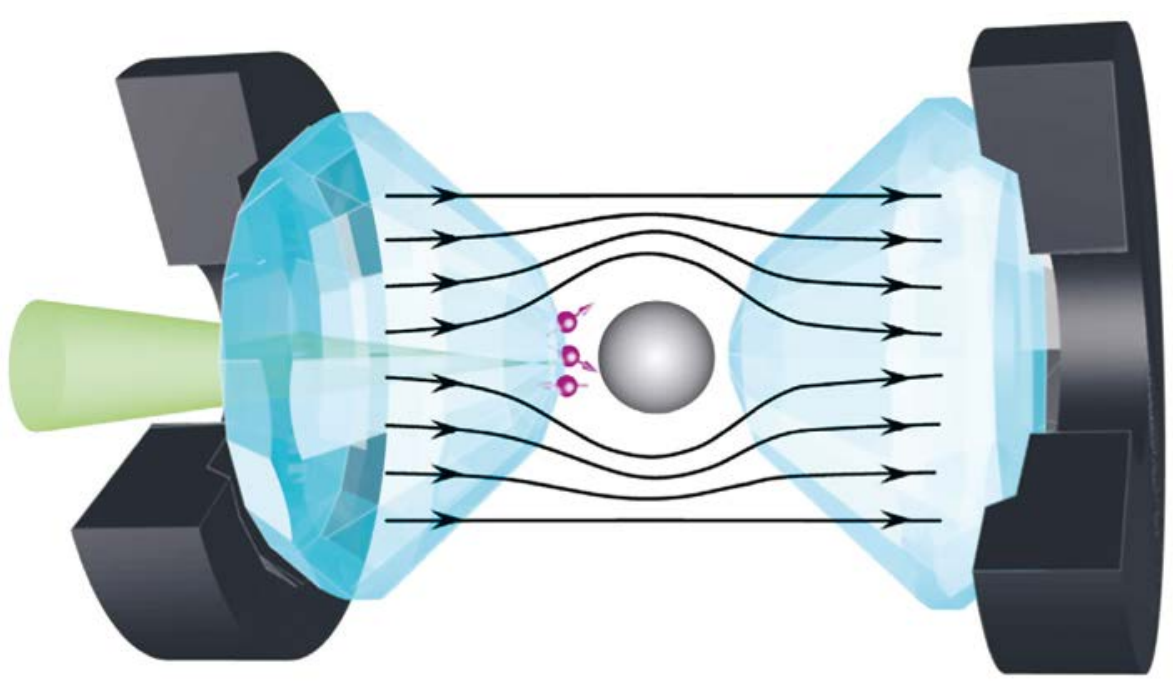

Figure 3: A high pressure cell is composed of two diamond anvils facing each other. The material under study $\left(\mathrm{MgB}_{2}\right)$, represented by the grey sphere, experiences a pressure of $7 \mathrm{GPa}$ inside the cell. A uniform external magnetic field is applied through the cell. The NV centers (pink spheres) implanted close to the surface of one of the anvils can measure the magnetic field inside the cell when pumped by a green laser beam sent through the anvil. For temperatures below $28 \mathrm{~K}$ at a pressure of $7 \mathrm{GPa}$, the $\mathrm{MgB}_{2}$ sample becomes superconductor and then produces a magnetic field that compensates exactly for the magnetic field inside it (Meissner effect). This induces a change in the overall magnetic field outside the sample that is measured by the NV centers. (Courtesy Lesik/Roch/ENS Paris-Saclay)

a single NV centre located a few nanometres below the surface. Such a small distance makes the NV centre sensitive to the magnetic field of a single nucleus, which can be detected using nuclear magnetic resonance techniques. This makes it possible to monitor the chemical shifts induced by neighbouring nuclei and to obtain information about the structure of the molecule. On the basis of similar techniques, a functional nuclear magnetic resonance spectrometer is currently being investigated. It would make it possible to develop a labon-a-chip for much more efficient drug analysis and improved medical diagnostics. Another medical application of NV centres is being developed within the framework of the METABOLIQS quantum flagship project. NV centres are used to efficiently polarise molecules used as markers in magnetic resonance imaging (MRI) devices. A gain of four orders of magnitude in the spin polarisation efficiency is expected. This would make it possible to manufacture MRI machines by an electrode deposited on the diamond surface and produce a current which can be directly used as a measurement signal. This opens up the possibility of developing much more compact and efficient devices, as the detector is directly deposited on the diamond crystal.

The NV centre in diamond is the most advanced example of a solid-state spin defect. However, there is a large class of other related defects which have very interesting characteristics. The silicon vacancy defect in diamond is another colour centre that can be used as an excellent source of photons for photonic integrated circuits. Tin vacancy and germanium vacancy are new colour centres currently under investigation. Other substrates such as silicon carbide or two-dimensional materials such as hexagonal boron nitride can host defects similar to the NV centre. In particular, silicon carbide defects have the interesting characteristic of emitting in the transparency window of optical fibres and are therefore well suited for telecommunication applications.

As we have shown previously, the NV centre in diamond and more generally solid-state spins are quantum objects with spectacular applications in the field of quantum sensing. In addition, they also hold great promise for quantum communications and quantum computing. We are only at the beginning of their application in a wide variety of fields. Many other applications, as yet unknown, are still to come and the future of these quantum sensors is very promising.

\section{REFERENCES}

[1] A. Beveratos, R. Brouri, T. Gacoin et al., Phys.Rev. Lett. 89, 187901 (2002)

[2] A. Gruber, A. Dräbenstedt, C. Tietz et al., Science 276, 2012 (1997).

[3] L. Thiel, Z. Wang, M. A. Tschudin et al., Science 364, 973 (2019).

[4] M. Lesik, T. Plisson, L. Toraille et al., Science 366, 1359 (2019).

[5] Quantum Technology | The future is Quantum (qt.eu) 